\title{
Magnon instability in ferromagnetic semiconductors under an intense electromagnetic field*
}

\author{
S. Coutinho \\ Departamento de Física, Universidade Federal de Pernambuco, 50.000 Recife, Pe, Brazil
}

L. C. M. Miranda

Departamento de Física, Universidade de Brasilia, 70.000 Brasilia, D. F. Brazil

(Received 18 February 1976)

\begin{abstract}
The magnon damping in an electron-magnon system in the presence of an electromagnetic wave is discussed. It is found that in the strong-field limit only multiphoton absorption processes are significant and as a result the magnon population grows with time. A numerical estimate of the actual growth rate is given for a typical ferromagnetic semiconductor like $\mathrm{CdCr}_{2} \mathrm{Se}_{4}$.
\end{abstract}

\section{INTRODUCTION}

It is well known that the electron-magnon interaction leads to the renormalization of the magnon spectrum in magnetic semiconductors and metals. ${ }^{1-3}$ At the same time, the electron-magnon interaction may constitute a fundamental mechanism in the damping of spin waves. It is therefore natural to expect that external fields changing the spectrum and occupation number of the electron states will influence the spectrum and damping of the spin waves. For instance, one knows that under the influence of a fairly strong dc electric field the magnon damping in ferromagnetic semiconductors may change considerably in magnitude and even in sign. The modification of the magnon damping and spectrum in constant uniform electric and magnetic fields was considered in previous papers. ${ }^{4-12}$ The motivation of these investigations was greatly enhanced by the development in the past few years of high-mobility ferromagnetic semiconductors like $\mathrm{Ag}$-doped $\mathrm{CdCr}_{2} \mathrm{Se}_{4}$.

In this paper one aims to discuss the modification of the spin-wave damping in an electron-magnon system in the presence of a classical electromagnetic wave. The quantum counterpart of this problem has been considered in Ref. 13. The electromagnetic field has frequency $\omega$ satisfying the inequalities

$$
c / v_{F} \gg \omega \tau \gg 1 \text {, and } \omega>\omega_{p},
$$

where $\tau$ is the electron relaxation time, $\omega_{p}$ is the plasma frequency, $v_{F}$ is the electron Fermi velocity, and $c$ is the speed of light. As for the spin waves we shall confine ourselves to those for which the wave number $k$ satisfies the condition

$$
k l \gg 1,
$$

where $l$ is the electron mean free path. Such magnons constitute well-defined elementary excitations of the system. Fulfillment of conditions (1) and (2) first ensures that the electromagnetic wave penetrates well into the sample and second means that the length of this wave is far greater than the electron mean free path and wavelength of the spin waves. Thus the spatial dependence of the field can be neglected and we need allow only for the dependence of $\vec{A}$ on time. The results so obtained are also applicable in the case of $\omega<\omega_{p}$, provided the length of the sample is much smaller than the depth of penetration of the field into the sample, $\delta=c\left(\omega_{p}^{2}-\omega^{2}\right)^{-1 / 2}$.

The main difference between the present theory and the one developed in Ref. 13 is twofold. In the first place, in the present paper we treat the electromagnetic field classically within the dipole approximation. Secondly, we will not make use of perturbation theory in the coupling of the interaction between the electrons and the electromagnetic field. It therefore follows that the present treatment is more adequate for studying the system at the strong radiation field regime.

Our model for a magnetic semiconductor is that of an interacting conduction-electron-localizedmoment system..$^{1-3}$ We describe the electron system by a parabolic conduction band and the local moment part by interacting (direct exchange) ionic moments $g \mu_{B} \vec{S}_{i}$ localized at lattice sites $\vec{R}_{i}$. The carriers and the localized moments are interacting by their exchange interaction which is taken to have the familiar $s-d$ contact form. The electron states are described by the solution to the Schrödinger equation for an electron in the field of a classical electromagnetic wave.

The magnon scattering by the electrons is treated using first-order perturbation theory, however, retaining the radiation field strength to all orders. The transition probabilities are then used to write a kinetic equation for the magnon population from which the damping rate is obtained. In the strongfield limit the electron-magnon collision involving multiphoton absorption becomes dominant, which 
in turn entails that the magnon population may become unstable.

\section{FORMULATION}

We assume a plane electromagnetic wave propagating along the $z$ axis (direction of the magnetization). The spatial dependence of the wave is neglected (dipole approximation). The time-dependent Schrödinger equation

$$
i \hbar \frac{\partial \psi}{\partial t}(\overrightarrow{\mathrm{x}}, t)=\frac{1}{2 m}\left|\frac{\hbar}{i} \vec{\nabla}-\frac{e}{c} \overrightarrow{\mathrm{A}}(t)\right|^{2} \psi(\overrightarrow{\mathrm{x}}, t),
$$

where

$$
\overrightarrow{\mathrm{A}}(t)=A_{0} \overrightarrow{\mathrm{e}}_{x} \cos \omega t
$$

has the solution (normalized in a box of volume $V$ )

$$
\psi(\overrightarrow{\mathrm{x}} t)=\frac{1}{\sqrt{v}} \exp \left(i \overrightarrow{\mathrm{p}} \cdot \overrightarrow{\mathrm{x}}-\frac{i}{2 m \hbar} \int^{t} d t^{\prime}\left|\hbar \overrightarrow{\mathrm{p}}-\frac{e}{c} \overrightarrow{\mathrm{A}}\left(t^{\prime}\right)\right|^{2}\right) .
$$

Here $\vec{p}$ is the electron wave vector such that in the absence of the radiation field the electron energy $E_{p}$ is $E_{p}=\hbar^{2} p^{2} / 2 m$.

In the case of a ferromagnetic semiconductor, however the conduction band becomes spin split such that the carrier energy is now given by ${ }^{1-3}$

$$
E_{p \sigma}=\hbar^{2} p^{2} / 2 m-\sigma J S / 2
$$

where $\sigma=+1$ for up carrier moments and $\sigma=-1$ for down moments. Here $J$ is the $s-d$ exchange parameter between the localized spins and the conduction electrons. As usual in Eq. (6) one is neglecting the Zeeman contribution since it is much smaller than $s-d$ splitting. Hence including the band splitting in Eq. (5) the electron wave function in a magnetic semiconductor under an electromagnetic plane wave is, therefore,

$$
\begin{gathered}
\psi_{\overrightarrow{\mathrm{p}} \sigma}(\overrightarrow{\mathrm{x}}, t)=\frac{1}{\sqrt{v}} \exp \left(\overrightarrow{\mathrm{p}} \cdot \overrightarrow{\mathrm{x}}-\frac{i}{2 m \hbar} \int^{t} d t^{\prime}\left|\hbar \overrightarrow{\mathrm{p}}-\frac{e}{c} \overrightarrow{\mathrm{A}}\left(t^{\prime}\right)\right|^{2}\right. \\
\left.+\frac{i}{2 \hbar} \sigma J S t\right) .
\end{gathered}
$$

Treating the electron-magnon interaction as a perturbation, the probability amplitude for the transition from state $1\left(\vec{p}_{1}, \sigma_{1}=+1\right)$ to state 2 $\left(\overrightarrow{\mathrm{p}}_{2}, \sigma_{2}=-1\right)$ due to the collision with a magnon of momentum $\hbar \overrightarrow{\mathrm{k}}$ is ${ }^{1-3}$

$$
\begin{aligned}
a(1-2 ; \overrightarrow{\mathrm{k}})=-\frac{i}{\hbar} \iint & d^{3} x d t \psi_{\overrightarrow{\mathrm{p}}_{2 \downarrow}}(\overrightarrow{\mathrm{x}}, t) M_{s d} \\
& \times e^{i\left(\overrightarrow{\mathrm{k}} \cdot \overrightarrow{\mathrm{x}}-\omega_{k} t\right)} \psi_{\overrightarrow{\mathrm{p}}_{1 \uparrow}}(\overrightarrow{\mathrm{x}}, t),
\end{aligned}
$$

where $M_{s d}=-J(S / 2 N)^{1 / 2}$ is the $s-d$ electron-magnon coupling with $N$ being the number of localized spins in the crystal of volume $V$. Substituting Eq.
(7) into Eq. (8) and performing the spatial integration we obtain

$$
\begin{aligned}
a(1-2 ; \overrightarrow{\mathrm{k}})= & -\frac{i M_{s d}}{\hbar} \frac{(2 \pi)^{3}}{v} \delta\left(\overrightarrow{\mathrm{p}}_{2}-\overrightarrow{\mathrm{p}}_{1}-\overrightarrow{\mathrm{k}}\right) \\
& \times \int d t e^{(i / \hbar)\left(E_{p_{2} \downarrow}-E_{p_{1} \uparrow}-\hbar \omega_{k}\right) t} \\
& \times e^{-i(\lambda / \hbar \omega) \sin \omega t},
\end{aligned}
$$

where $\lambda=e \hbar \overrightarrow{\mathrm{k}} \cdot \overrightarrow{\mathrm{E}}_{0} / m \omega$ is the field parameter. The integration over $t$ can easily be performed by expanding $\exp [-(i \lambda / \hbar \omega) \sin \omega t]$ as a series in Bessel functions so that one gets

$$
\begin{aligned}
a(1 \rightarrow 2 ; \overrightarrow{\mathrm{k}})= & -2 \pi i M_{s d}\left(\frac{2 \pi}{v}\right)^{3} \delta\left(\overrightarrow{\mathrm{p}}_{2}-\overrightarrow{\mathrm{p}}_{1}-\overrightarrow{\mathrm{k}}\right) \\
& \times \sum_{\nu=-\infty}^{+\infty} J_{\nu}\left(\frac{\lambda}{\hbar \omega}\right) \delta\left(E_{p_{2 \downarrow}}-E_{p_{1 \uparrow}}-\hbar \omega_{k}-\nu \hbar \omega\right) .
\end{aligned}
$$

Here $J_{\nu}(\lambda / \hbar \omega)$ is the Bessel function of order $\nu$. From the well-known relation between the scattering amplitude and the $T$ matrix ${ }^{14,15}$ we can then use Eq. (10) to obtain the transition probability per unit time $T_{\nu}(1-2 ; \overrightarrow{\mathrm{k}})$ for the transition from state $1\left(\overrightarrow{\mathrm{p}}_{1}, \sigma_{1}=+1\right)$ to a state $2\left(\overrightarrow{\mathrm{p}}_{2}=\overrightarrow{\mathrm{p}}_{1}+\overrightarrow{\mathrm{k}}, \sigma_{2}=-1\right)$ due to a collision with a magnon $\overrightarrow{\mathrm{k}}$ with absorption $(\nu>0)$ or emission $(\nu<0)$ of $|\nu|$ photons. One gets

$$
\begin{aligned}
T_{\nu}(1-2 ; k)= & \frac{2 \pi}{\hbar} M_{s d}^{2} J_{\nu}^{2}\left(\frac{\lambda}{\hbar \omega}\right) \\
& \times \delta\left(E_{p_{2 \downarrow} \downarrow}-E_{p_{1 \uparrow}}-\hbar \omega_{k}-\nu \hbar \omega\right) .
\end{aligned}
$$

Since we are treating the electromagnetic field classically the number of photons $|\nu|$ has the same meaning as in the theory of bounded systems under strong laser fields $s^{15,16}$-it is the number of $\hbar \omega$ such that $\Delta E=E_{2}-E_{1}-\hbar \omega_{k}=\nu \hbar \omega$.

Finally, the rate of change of the number of magnons of wave number $k, d N_{k} / d t$, is then given in terms of the transition probability $T_{\nu}$ as $^{17,18}$

$$
\frac{d N_{k}}{d t}=\gamma_{k} N_{k}
$$

where

$$
\begin{aligned}
\gamma_{k}=\frac{2 \pi}{\hbar} M_{s d}^{2} \sum_{\nu=-\infty}^{+\infty} \sum_{\overrightarrow{\mathrm{p}}} & J_{\nu}^{2}\left(\frac{\lambda}{\hbar \omega}\right)\left(f_{p+k \downarrow}-f_{p \uparrow}\right) \\
& \times \delta\left(E_{p+k \downarrow}-E_{p \uparrow}-\hbar \omega_{k}-\nu \hbar \omega\right) .
\end{aligned}
$$

In Eq. (13), $f_{p \sigma}$ is the Fermi distribution function. If $\gamma(k)>0$ the magnon population grows with time, whereas for $\gamma(k)<0$ we have damping. Actually the criterion for the onset of the instability is somewhat more involved. In our discussion so far we have completely neglected other mecha- 
nisms such as multimagnon, magnon-phonon, etc., which may interact with the magnons and lead to a finite magnon lifetime even in the absence of the electron-magnon interaction. We may take them into account by introducing a phenomenological magnon decay rate $\nu(k)$ due to other processes than magnon emission and absorption by electrons. The actual criterion for the magnon instability for a particular wave vector $\vec{k}$ is therefore

$$
\gamma(k)-\nu(k)>0
$$

\section{GROWTH RATE}

In this section we shall calculate $\gamma(k)$ in the limit of strong radiation field and show that under certain circumstances $\gamma(k)$ may be positive and larger than $\nu(k)$. The weak-field limit, $\lambda \ll h \omega$, is essentially discussed in Refs. 5 and 19.

In the strong-field limit, $\lambda \gg h \omega$ and the argument of the Bessel function in Eq. (13) is large. Of course $\lambda \gg \hbar \omega$ depends upon the direction of $\overrightarrow{\mathrm{k}}$. However, since we shall be mainly interested in $\overrightarrow{\mathrm{k}}$ parallel to $\overrightarrow{\mathrm{E}}_{0}$ the condition for $\lambda \gg \hbar \omega$ is essentially $E_{0}$ large. For large values of argument, the Bessel function is small except when the order is equal to the argument. The sum over $\nu$ in Eq. (13) may then be written approximately as

$\sum_{\nu=-\infty}^{+\infty} J_{\nu}^{2}\left(\frac{\lambda}{\hbar \omega}\right) \delta(E-\nu \hbar \omega)=\frac{1}{2}[\delta(E-\lambda)+\delta(E+\lambda)]$.

The factor $\frac{1}{2}$ may be verified by integrating both sides of the equation with respect to $E=E_{p+k \downarrow}$ $-E_{p \uparrow}-\hbar \omega_{k}$. The first $\delta$ function corresponds to the emission and the second to the absorption of $\lambda / \hbar \omega$, only multiphoton processes are significant. In other words, for the strong-field case, the electron-magnon collision takes place with the absorption or emission of $\lambda / \hbar \omega \gg 1$ photons.

Substituting Eq. (15) in Eq. (13) the magnon damping becomes

$$
\begin{aligned}
\gamma(k)=\frac{\pi M_{s d}^{2}}{\hbar} \sum_{p}( & \left.f_{p+k \downarrow}-f_{p \uparrow}\right) \\
\times & {\left[\delta\left(E_{p \nmid k \downarrow}-E_{p \uparrow}-\hbar \omega_{k}-\lambda\right)\right.} \\
& \left.+\delta\left(E_{p+k \downarrow}-E_{p \uparrow}-\hbar \omega_{k}+\lambda\right)\right] .
\end{aligned}
$$

If we further assume that $\lambda \gg E_{F}$ we may neglect the contribution of the process in which photons are emitted compared to the contribution of processes in which photons are absorbed. This means that in the case of $\lambda \gg E_{F}$ the contribution of the first $\delta$ function may be neglected so that Eq. (16) reduces to

$$
\begin{array}{rl}
\gamma(k)=\frac{V M_{s d}^{2}}{4 \pi \hbar} \int_{0}^{\infty} d p p^{2} \int_{-1}^{+1} & d x\left[f\left(E_{p \uparrow}+\hbar \omega_{k}-\lambda\right)-f\left(E_{p \uparrow}\right)\right] \\
& \times \delta\left(E_{p+k \downarrow}-E_{p \uparrow}-\hbar \omega_{k}+\lambda\right)
\end{array}
$$

after transforming the sum over $\vec{p}$ into an integral; here $x$ denotes the cosine of the angle between $\vec{p}$ and $\vec{k}$ where the polar axis for the $\vec{p}$ integration was taken along the direction of $\vec{k}$.

The criterion for $\gamma(k)$ to be positive can now, be established. By recalling that $f(E)>f\left(E^{\prime}\right)$ when $E \leq E^{\prime}$ it is easy to see that $\gamma(k)$ is positive if

$$
\lambda-\hbar \omega_{k}>0 \text { or } \overrightarrow{\mathrm{k}} \cdot \overrightarrow{\mathrm{v}}_{0}-\omega_{k}>0,
$$

where $\overrightarrow{\mathrm{v}}_{0}=e \overrightarrow{\mathrm{E}}_{0} / m \omega$. Hence, for a particular magnon wave vector the necessary condition for the onset of the magnon instability is just the Cerenkov condition $\overrightarrow{\mathrm{k}} \cdot \overrightarrow{\mathrm{v}}_{0}>\omega_{k}$.

Approximating $f\left(E_{p \sigma}\right)$ by a step function and performing the integration in Eq. (17) one finally gets

$$
\gamma(k)=\frac{m^{2} V M_{s d}^{2}}{4 \pi \hbar^{4} k}\left(\frac{e \overrightarrow{\mathrm{k}} \cdot \overrightarrow{\mathrm{E}}_{0}}{m \omega}-\omega_{k}\right),
$$

provided $\lambda \gg E_{F}$. The above result is formally analogous to the one of the electron-magnon system in the presence of a dc electric field. The difference lies on the fact that in the later case $\overrightarrow{\mathrm{v}}_{0}=e \overrightarrow{\mathrm{E}}_{0} / m \omega$ is replaced by $\overrightarrow{\mathrm{v}}_{d} \cdot{ }^{4-7}$

The condition $\lambda \gg E_{F}$ restricts the present theory only to high values of $k$. To see this let us consider in more details the condition $\lambda \gg E_{F}$. For simplicity we shall consider only magnons propagating parallel to $\overrightarrow{\mathrm{E}}_{0}$. For a given $k$ the condition $\lambda=E_{F}$ defines a critical field strength $E_{c}$ given by

$$
E_{c}=m \omega E_{F} / e \hbar k,
$$

from which we have

$$
I_{c}=\frac{1}{8 \pi \alpha \hbar}\left(\frac{m \omega E_{F}}{\hbar k}\right)^{2}
$$

for the minimum laser intensity such that $\lambda=E_{F}$; here $\alpha=e^{2} / \hbar c$. To get a numerical estimate of the value of $I_{c}$ let us consider the following values for the physical parameters of a typical highmobility magnetic semiconductor like $\mathrm{CdCr}_{2} \mathrm{Se}_{4}$ doped with ${ }^{20-23} \mathrm{Ag}: J_{s u}=10^{-14} \mathrm{erg}, S=\frac{3}{2}, N / V=10^{23}$ $\mathrm{cm}^{-3}, E_{F}=1.2 \times 10^{-13} \mathrm{erg}$. Using a Nd:glass laser $(\lambda=1.06 \mu)$ which is capable to provide a range of power densities from $50 \mathrm{~W} / \mathrm{cm}^{2}$ to beyond $10^{10}$ $\mathrm{W} / \mathrm{cm}^{2}$, it can be seen that $I_{c}$ becomes

$$
I_{c}=2.4 \times 10^{23} \mathrm{k}^{-2} \mathrm{~W} / \mathrm{cm}^{2} .
$$

Hence for $k=10^{7} \mathrm{~cm}^{-1}, I_{c}$ is typically of the order of $10^{9} \mathrm{~W} / \mathrm{cm}^{2}$. For $k=10^{6} \mathrm{~cm}^{-1}$ the power needed is two orders of magnitude higher which already starts to impose some technical difficulties, although via passive optical techniques one may eventually reach power densities of about $10^{11}$ $\mathrm{W} / \mathrm{cm}^{2}$.

Therefore, restricting ourselves to high values 
of $k$ and using, for instance, a Nd:glass laser with a power density of the order of $10^{10} \mathrm{~W} / \mathrm{cm}^{2}$ together with the values of the physical parameters given above we estimate $\gamma(k)$ to be of the order of $10^{7} \mathrm{sec}^{-1}$ for $k=10^{7} \mathrm{~cm}^{-1}$. One notes that under the above conditions $V_{0}=e E_{0} / m \omega$ is of the order of $2.7 \times 10^{7} \mathrm{~cm} \mathrm{sec}^{-1}$ which is much greater than $v_{s}=\omega_{k} / k \simeq 10^{5} \mathrm{~cm} \mathrm{sec}^{-1}$.

So far we have completely neglected the spinwave losses $\nu(k)$ due to order processes. As mentioned before the actual condition for a net amplification requires the magnon growth to be greater than the losses [Eq. (14)]. The magnon linewidth data available for $\mathrm{CdCr}_{2} \mathrm{Se}_{4}$ from parallel pumping measurements ${ }^{23}$ leads us to take $\nu \sim 4$ $\times 10^{6} \mathrm{sec}^{-1}$. Hence, since the amplification factor $\gamma(k)$ may be of the order or greater than the losses $\nu(k)$ one might hope to achieve a net amplification of spin waves of high $k$ 's when the system is under an intense laser field. This is of particular interest since the conventional amplification of spin waves via a dc electric field $d^{4-8}$ favors magnons of relatively small $k$ 's, i.e., $k=10^{5} \mathrm{~cm}^{-1}$.

\section{CONCLUSIONS}

In this paper we have considered an electronmagnon system in the field of an intense electromagnetic wave. It was found that in the strongfield limit, $\lambda \gg E_{F}$, only the electron-magnon collisions accompanied by multiphoton absorption are significant, and as a result the magnon population may grow with time when the field strength $E_{0}$ exceeds certain threshold value given by Eqs.
(14) and (20). Furthermore, it was also shown that the present theory is restricted to high values of $k$. The reason why the present mechanism is limited to high $k$ is essentially due to the condition $\lambda \gg E_{F}$ which is only satisfied, from a practical point of view, for high $k$.

Physically the magnon instability in the field of a strong electromagnetic wave may be understood by assuming that the effect of the strong field is to give a drift velocity $v_{0}=e E_{0} / m \omega$ to the carriers. Hence when $v_{0}$ exceeds the phase velocity of the spin waves the magnon population in the Cerenkov cone may in principle grow with time. The actual observation of the population growth is achieved only if $\gamma(k)$ is greater than $\nu(k)$. A numerical estimate of $\gamma(k)-\nu(k)$ based upon the values of the physical parameters of $\mathrm{CdCr}_{2} \mathrm{Se}_{4}$ doped with $\mathrm{Ag}$ suggests us that using a Nd:glass laser with a power density of about $10^{10} \mathrm{~W} / \mathrm{cm}^{2}$ one might in principle observe a net amplification of spin waves.

In conclusion it should be emphasized that our model contains a number of simplified assumptions. Nevertheless, some essential conclusions can be drawn therefrom. Amongst them, the present mechanism has the ability of exciting spin waves of high $k$ propagating perpendicular to the $z$ axis (i.e., parallel to $\overrightarrow{\mathrm{E}}_{0}$ ). This is of particular interest since the conventional methods (e.g., drifting carries ${ }^{4-8}$ ) favors amplification along the $z$ axis. Secondly, since the growth rate is proportional to $J^{2}$ it could be used as a new tool for the investigation of the $s-d$ exchange parameter.
*Work partially supported by the Brazilian Agencies CNPq (Conselho Nacional de Pesquisas), CAPES (Campanha de Aperfeiçoamento de Pessoal de Ensiño Superior), and BNDE (Banco Nacional de Desenvolvimento Econômico).

${ }^{1}$ R. B. Woolsey and R. M. White, Phys. Rev. B $\underline{1}, 4474$ (1970).

${ }^{2}$ S. Methfessel and D. C. Mattis, in Handbuch der Physik, edited by S. Flügge (Springer-Verlag, Berlin, 1962), Vol. XVIII/1.

${ }^{3}$ S. V. Vonsovski and Yu.A. Yzyumov, Usp. Fiz. Nauk. 78,3 (1963) [Sov. Phys.-Usp. 5,723 (1963)].

${ }^{4}$ M. D. Coutinho, Jr., L. C. M. Miranda, and S. M. Rezende, Phys. Status Solidi B 57, 85 (1973).

${ }^{5}$ L. C. M. Miranda, Phys. Status Solidi B 60, 617 (1973).

${ }^{6}$ M. D. Coutinho, Jr., L. C. M. Miranda, and S. M. Rezende, Phys. Status Solidi B 65, 689 (1974).

${ }^{7}$ M. D. Coutinho, Jr., L. C. M. Miranda, and S. M. Rezende, Phys. Status Solidi B 66, 395 (1974).

${ }^{8}$ M. D. Coutinho, Jr., L. C. M. Miranda, and S. M. Rezende, Rev. Brasil. Fis. 4, 399 (1974).

${ }^{9}$ S. Coutinho and L. C. M. Miranda, Il Nuovo Cimento B 28, 161 (1975).
${ }^{10}$ L. C. M. Miranda, Solid State Commun. 16, 973 (1975).

${ }^{11}$ M. A. F. Gomes and L. C. M. Miranda, Phys. Rev. B 12, 3788 (1975).

${ }^{12}$ L. C. M. Miranda, Phys. Rev. B 13, 3180 (1976).

${ }^{13}$ S. Coutinho and L. C. M. Miranda, Prog. Theor. Phys. 54, 927 (1975).

${ }_{14}$ P. Roman, Advanced Quantum Mechanics (AddisonWesley, Reading, Mass., 1965), p. 285.

${ }^{15}$ H. Reiss, Phys. Rev. A 1, 803 (1970).

${ }^{16}$ H. Reiss, Phys. Rev. D $\overline{4}, 3533$ (1971).

${ }^{17}$ F. G. Harris, in Advances in Plasma Physics, edited by A. Simon and W. B. Thompson (Addison-Wesley, Reading, Mass., 1969), Vol. 3.

${ }^{18} \mathrm{D}$. Pines, The Many-Body Problem (Benjamin, New York, 1962).

${ }^{19}$ L. C. M. Miranda, Phys. Rev. B 12, 5075 (1975).

${ }^{20}$ I. Balderg and H. L. Pinch, Phys. Rev. Lett. 28, 909 (1972).

${ }^{21}$ H. W. Lehmann, Phys. Rev。 163, 488 (1967).

${ }^{22}$ R. L. LeCraw, H. von Philipsborn, and M. D. Sturge, J. Appl. Phys. 38, 965 (1967).

${ }^{23}$ R. Bartknowski, J. S. Page, and R. L. LeCraw, J. Appl. Phys. 39, 1071 (1968). 